\title{
Big Data Analytics for Earth Sciences: the EarthServer approach
}

\author{
Peter Baumann ${ }^{\mathrm{a}, \mathrm{b} *}$, Paolo Mazzetti ${ }^{\mathrm{c}}$, Joachim Ungar ${ }^{\mathrm{d}}$, Roberto Barbera ${ }^{\mathrm{e}, \mathrm{f}, \mathrm{g}}$, \\ Damiano Barboni ${ }^{\text {h }}$, Alan Beccati ${ }^{\mathrm{a}}$, Lorenzo Bigaglic ${ }^{\mathrm{c}}$, Enrico Boldrinic ${ }^{\mathrm{c}}$, Riccardo Bruno ${ }^{\mathrm{e}, \mathrm{f}}$, \\ Antonio Calanducci ${ }^{f}$, Piero Campalani ${ }^{a}$, Oliver Clementsi, Alex Dumitru ${ }^{a}$, Mike Granti, \\ Pasquale Herzigj, George Kakaletris ${ }^{k}$, John Laxton', Panagiota Koltsidak, Kinga \\ Lipskoch $^{\mathrm{a}}$, Alireza Rezaei Mahdiraji ${ }^{\mathrm{a}}$, Simone Mantovani ${ }^{\mathrm{h}}$, Vlad Merticariua, \\ Antonio Messina ${ }^{m}$, Dimitar Miseva, Stefano Natali ${ }^{\text {h }}$, Stefano Nativic ${ }^{\mathrm{c}}$, Jelmer Oosthoek ${ }^{\mathrm{a}}$, \\ Marco Pappalardo ${ }^{m}$, James Passmore ${ }^{n}$, Angelo Pio Rossi ${ }^{a}$, Francesco Rundo ${ }^{e}$, \\ Marcus Sen ${ }^{n}$, Vittorio Sorbera ${ }^{\mathrm{e}}$, Don Sullivan ${ }^{\circ}$, Mario Torrisi ${ }^{\mathrm{f}}$, Leonardo Trovato $^{\mathrm{m}}$, \\ Maria Grazia Veratelli ${ }^{\text {h }}$ and Sebastian Wagner ${ }^{\mathrm{j}}$ \\ ${ }^{a}$ Large-Scale Scientific Information Systems, Jacobs University, Bremen, Germany; ${ }^{b}$ Rasdaman \\ $\mathrm{GmbH}$, Bremen, Germany; 'CNR-IIA, National Research Council of Italy, Institute of Atmospheric \\ Pollution Research, Florence, Italy; ${ }^{d}$ EOX IT Services GmbH, Vienna, Austria; ${ }^{e}$ Consorzio COMETA, \\ Catania, Italy; fDivision of Catania, Italian National Institute for Nuclear Physics, Catania, Italy; \\ ${ }^{g}$ Department of Physics and Astronomy, University of Catania, Catania, Italy; ${ }^{h}$ MEEO S.r.l., \\ Ferrara, Italy; iPlymouth Marine Laboratory, Plymouth, UK; ${ }^{j}$ Fraunhofer IGD, Darmstadt, \\ Germany; 'Athena Research and Innovation Center in Information Communication \&Knowledge \\ Technologies, Athens, Greece; 'British Geological Survey, Edinburgh, UK; ${ }^{m}$ Software Engineering \\ Italia S.r.I., Catania, Italy; ${ }^{n}$ British Geological Survey, Keyworth, UK; ${ }^{\circ}$ NASA Ames Research \\ Center, Moffett Field, CA, USA
}

Big Data Analytics is an emerging field since massive storage and computing capabilities have been made available by advanced e-infrastructures. Earth and Environmental sciences are likely to benefit from Big Data Analytics techniques supporting the processing of the large number of Earth Observation datasets currently acquired and generated through observations and simulations. However, Earth Science data and applications present specificities in terms of relevance of the geospatial information, wide heterogeneity of data models and formats, and complexity of processing. Therefore, Big Earth Data Analytics requires specifically tailored techniques and tools. The EarthServer Big Earth Data Analytics engine offers a solution for coverage-type datasets, built around a high performance array database technology, and the adoption and enhancement of standards for service interaction (OGC WCS and WCPS). The EarthServer solution, led by the collection of requirements from scientific communities and international initiatives, provides a holistic approach that ranges from query languages and scalability up to mobile access and visualization. The result is demonstrated and validated through the development of lighthouse applications in the Marine, Geology, Atmospheric, Planetary and Cryospheric science domains.

Keywords: big data; Big Data Analytics; array databases; Earth Sciences; interoperability; standards 


\section{Introduction}

In the recent years, the evolution of communication and digital storage technologies allowed the collection of a huge amount of information raising the need for effective ways of maintaining, accessing, and processing data efficiently. In this context, the term 'big data' became widely used. Its first definition, by Doug Laney of META Group (then acquired by Gartner; Laney 2001), as data requiring high management capabilities characterized by the $3 \mathrm{Vs}$ : Volume, Velocity and Variety, is still relevant, especially for the geospatial data domain. It points out that big data does not simply mean large datasets (big Volume) but also efficient dataset handling (big Velocity) and great heterogeneity (big Variety). Later, other Vs have been added by other authors: Veracity (i.e. addressingquality and uncertainty), Value, etc.

In the scientific domain, several disciplinary areas are facing big data challenges as part of an innovative approach to science usually referred to as e-Science. Earth Sciences have been some of the disciplinary domains most strongly pushing, and potentially benefiting from, the e-Science approach, intended as 'global collaboration in key areas of science, and the next generation of infrastructure that will enable it' (Hey and Trefethen 2002). They were in the forefront in many initiatives on distributed computing trying to realize the e-Science vision, including high performance computing, grid technologies (Petitdidier et al. 2009), and cloud services. The reason is that Earth Sciences raise significant challenges in terms of storage and computing capabilities, as:

(1) They encompass a wide range of applications: from disciplinary sciences (e.g. Climate, Ocean, Geology) to the multidisciplinary study of the Earth as a system (the so-called Earth System Science). Therefore, Earth Sciences make use of heterogeneous information (Big Variety):

(a) covering a diverse temporal range (such as for Climate and Geological studies);

(b) supporting a wide spatial coverage (the whole Earth, for global studies, and beyond when considering planetary sciences);

(c) modeling many different geospatial data types, including profiles, trajectories, regularly and irregularly gridded data, etc.;

(2) They are based on observations and measurements coming from in situ and remote- sensing data with ever-growing spatial, temporal, and radiometric resolution, requiring handling of Big Volumes, e.g. Sentinel satellites will increase the size of the ESA data archive to more than 20 PB in 2020 (Houghton 2013).

(3) They make use of complex scientific modeling and simulations to study complex scenarios (e.g. for Climate Change) requiring fast processing (Big Velocity).

It is therefore clear that - referring to the Big data Vs - big Volume, big Variety, and high Velocity are characteristic issues of Earth Science data systems.

The work presented in this paper is result of the project EarthServer funded under the European Community's Seventh Framework Programme in 2011-2014. EarthServer is coordinated by Jacobs University of Bremen, with the participation of European research centers and private companies, and with an international collaboration with NASA. The project objective is the development of specific solutions for supporting open access and ad hoc analytics on Earth Science (ES) big data, based on the OGC geoservice standards. EarthServer included research and development activities to develop client and server technologies, and demonstration activities through a set of lighthouse applications for validation. 
The paper presents and discusses the main outcomes of the project with contributionsfrom the different research groups involved.

\section{Big Data Analytics challenges for Earth Sciences}

Since its beginning, the EarthServer project paid great attention to the collection of scientific and technological requirements from relevant Earth Science communities. Moreover, a specific action was dedicated to the collection of requirements and evaluation of the alignment with international initiatives on Earth and environmental data-sharing like GEOSS (http://www.earthobservations.org), INSPIRE (http://inspire.ec. europa.eu), and Copernicus (http://www.copernicus.eu).

The EarthServer project addressed the scientific communities through partners that are part of the communities themselves. This approach was successful because it greatly simplified the interaction. The Plymouth Marine Laboratory (PML) acted as a proxy toward the Marine community, as the British Geological Survey (BGS) did for the Solid Earth community and Meteorological Environmental Earth Observation S.r.I. (MEEO) for the Atmospheric community. They collected requirements and validated the ongoing activities, through questionnaires, consultations, and organizations of dedicated work- shops, usually back-to-back with relevant events for the community. In addition, the Earth Science community as a whole was addressed through dedicated meetings held during the annual European Geosciences Union (EGU) General Assembly.

Other actions were specifically directed to the Earth Observation community through the organization of presentation and meetings during the Group on Earth Observation (GEO) Plenary and the co-organization of the ESA 'Big Data from Space' conference (Bargellini et al. 2013).

The main results of this activity can be summarized in the following list of general requirements (Mazzetti et al. 2013):

(1) Earth Observation applications are already facing the Big Data issue, with a need for advanced solutions supporting big data handling and big data analytics.

(2) There is a need for flexible solutions enabling ad hoc analytics on big data forscientific data exploration on demand.

(3) Users require big data technologies supporting multiple data models and reducingdata transfer.

(4) Users require advanced visualization techniques easily integrated in differentGUIs including Web and mobile systems.

\section{The EarthServer approach}

\section{General approach}

In a nutshell, EarthServer provides open, interoperable, and format-independent access to 'Big Geo Data', ranging from simple access and extraction to complex agile analytics/ retrieval services on data. An Array Database, rasdaman (http://www.rasdaman.org) (Jacobs University Bremen and rasdaman GmbH 2013; Rasdaman, 2013), empowers the EarthServer technology to integrate data/metadata retrieval, resulting in same level of search, filtering, and extraction convenience as is typical for metadata (Array DBMS 2014). Traditionally, data dealing with the Earth or with planetary systems are categorized into vector, raster, and metadata. The latter is 
what is generally considered small, semantic-rich, and queryable. Vector data representing points, lines, areas, etc. have reached this Holy Grail since some time, too. Raster data - i.e. data points aligned on some regular or irregular grid - due to their sheer size are generally considered as suitable only for download, maybe extracting subsets, but otherwise with no particular queryable semantics and without a standard functionality set. Standardization is one of the means to enhance interoperability. In the geospatial data domain, the Open Geospatial Consortium

(OGC) plays a key role in standardization. Concerning service interfaces, it provides specifications for accessing different data: the Web Feature Service (WFS) is tailored to serve vector data, while the Web Coverage Service (WCS) is devoted to multidimensional raster data, point clouds, and meshes; the Web Map Service (WMS) has a special role in that it aims at visualizing vector and raster maps in 2D in the simplest fashion possible. WCS is particularly interesting for data archives since it allows accessing data values for further processing and not just for visualization (as WMS does). One use case is to obtain data in guaranteed unmodified, such as bathymetry data; another one is serverside processing to obtain the tailor-made product required. Part of this further processing can be conveniently implemented server-side, directly within the archive and executed upon request. The Web Coverage Processing Service (WCPS) is specifically designed to enhance data archives by doing this, providing 'analytics' directly on top of them, aimingat enhancing data use and exploitation.

For spatio-temporal 'Big Data', the OGC has defined its unified coverage model (nicknamed GMLCOV) which refines the abstract model of ISO 19123 (ISO 2005) to a concrete, interoperable model that can be conformance tested down to single pixel level. Coverage is a subtype (i.e., specialization) of a feature, a feature being a geographic object; informally speaking, coverage is a digital representation of some space-time (multidimensional) varying phenomenon (Baumann 2012a). Technically, a coverage encompasses regular and irregular grids, point clouds, and general meshes. As this notion is not tied to a particular service model, many services can receive or generate coverages, such as OGC WFS, WMS, WCS, WCPS, and WPS. Specifically, the Web Coverage Service standard provides rich, tailored functionality essential for data access and analysis. For the latter, WCS is closely connected to the Web Coverage Processing Service (WCPS) which defines a query language on coverages, currently on spatio- temporal rasters, i.e. geo-referenced arrays addressable by some spatiotemporal coordinate reference system. Generally speaking, this includes n-D sensor, image, simulation output, and statistics data. Over such multidimensional data entities, the WCPS standard offers a leap ahead with respect to interoperable processing: it defines a powerful and flexible query language that, on top of data archives, enables coverage data to be used in complex queries. Derived products can thus be built on the fly and combined with other coverages.

EarthServer recognizes that Big Data in geoservices often means coverages certainly with regard to volume, but generally in all respects of the Vs characterizing Big Data. Therefore, the whole architecture centers around supporting coverages. The nucleus is the rasdaman array database serving regular and irregular grids and, experimentally, point clouds. It is particularly suitable for geoservices that are intensive in both data quantity and processing because its query language gives the flexibility to phrase any task without reprogramming the server (as is effectively the case, e.g. with WPS-based systems).

The OGC standards for Big Geo Data, centered around the OGC coverage data and service model, represent a suitable client/server interface for general purpose use in the 
Earth Sciences. Therefore, the WCS and WCPS standards have been adopted by EarthServer, together with WMS for 2D visualization. EarthServer extends this platform toward a comprehensive coverage analytics engine. It comprehensively supports the WMS/WCS/WCPS suite on multidimensional, spatio-temporal coverages; data and metadata search has been integrated, thereby effectively abolishing this age-old distinction; interfaces to GIS tools like MapServer and GDAL have been established; the server engine has been massively parallelized to achieve strong scalability; and 3D browser clients have been established effectively hiding the query language from casual users while allowing query writing for expert users. This technology forms the common platform for the six Lighthouse Applications which together comprehensively address theEarth sciences.

Finally, findings obtained in platform development and service operation are fed back into the standardization process where they have significantly shaped recent WCS and WCPS specification work.

\section{Data service infrastructure}

Figure 1 shows a UML component diagram of the overall EarthServer architecture from the data flow and access perspective. Details of the single components are omitted and the high-level elements are connected by dependencies on elements which they drive (as is the case with the ingestion system) or from which they access stored data. The external interfaces are also highlighted in the left side of the 'EarthServer' data service package.

The infrastructure components and their main functions are described in the following subsections.

\section{Data layer and file based ingestion}

Depending on the specific service provider, data can be accessed as a network resource or can be stored locally on internal file servers. Regardless of the source type and location, the first required step is the data ingestion into rasdaman. Basically, it means providing the rasdaman server with descriptive information about the dataset so that it can be properly accessed by the rasdaman array database engine and translated into a GMLCOV instance for delivery. Data ingestion is performed through a set of command line tools.

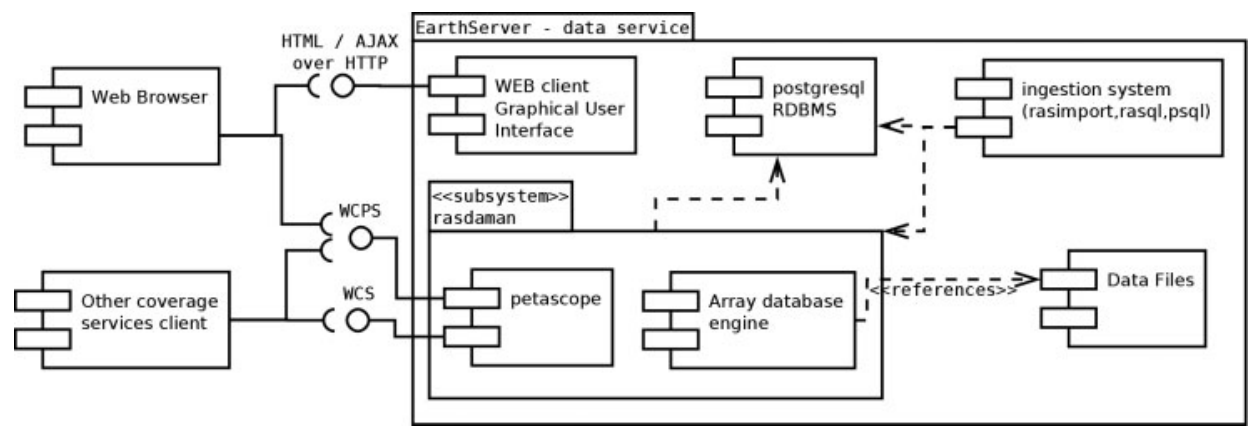

Figure 1. EarthServer data service component diagram (generic architecture). 


\section{Backend: Rasdaman (structured array and in situ)}

The rasdaman system is a so-called array DBMS, a recent research direction in databases which it actually pioneered. An array DBMS offers the same quality of service - such as query language, optimization, parallel and distributed query processing - on large multidimensional arrays as conventional SQL systems offer on sets (Array DBMS 2014; Baumann et al. 2011).

The conceptual model of rasdaman consists of multidimensional arrays with some cell type and n-D extent. The rasdaman query language, rasql (Rasdaman GmbH 2013), adds generic array operators which can be combined freely. Its expressiveness encompasses subsetting, cell manipulation, and general image, signal, and statistical analysis up to, e.g., the Fourier Transform. As it will be discussed in the Related Work section, rasdaman currently is the only Array DBMS operationally used on multi-TB holdings, fully parallelized, and proven in scalability.

The storage model relies on the partitioning of the arrays into sub-arrays. Array partitioning for speeding up access exists in data formats such as TIFF and NetCDF. In the service communities, this concept has been described in the context of rasdaman under the name 'tiling' (Baumann 1994), later it also has been termed 'chunking' (Sarawagi and Stonebraker 1994). Actually, chunking often refers to a regular partitioning while the rasdaman tiling covers the spectrum of regular, irregular, and non-aligned partitioning in any number of dimensions. As opposed to, say, PostGIS Raster (http:// postgis.net/docs/manual-2.1/using_raster_dataman.html), tiling is transparent to the user, but accessible to the database tuner as an optimization method; for example, for time series analysis tiles would be stretched along time while having a smaller spatial footprint, thereby reducing disk accesses during query evaluation. Storage of tiles is either in a relational database (RDBMS) or in flat files. An RDBMS - in EarthServer this is PostgreSQL - offers the advantage of information integration with metadata plus the wealth of tools available, but comes at some extra cost, e.g. due to the data duplication as well as for transaction handling. File-based storage allows access to data in the pre-existing archive, which is faster and not relying on redundant storage in a database, but lacks transaction support; additionally, existing archives frequently are not tuned toward user access patterns. Therefore, in practice often a mix will be optimal.

The rasdaman engine as such operates on arrays in a domain-agnostic way. The specific geo-semantics of coordinates, regular and irregular grids, etc., are provided by an additional layer in the rasdaman overall architecture, called petascope, which is describedin the following.

\section{Rasdaman web service interfaces (petascope)}

Coverages offered by the data service are made accessible over the web by the petascope component of rasdaman, which is also the reference implementation of the WCPS standard (Aiordachioaie and Baumann 2010). Petascope consists of Java servlets that leverage several open source geospatial and geometry libraries, as well as rasdaman data access libraries and relational database access components. Basically, it translates incoming processing requests (WCPS queries) into rasdaman (rasql) queries to efficiently fetch and process array data (according to information in the coverage rangeType and domainSet elements). It then translates the output into the proper coverage type, formatted according to the requested encoding. Moreover, the 'encode' WCPS operator allows for delivering coverage data in other formats (not necessarily maintaining all 
coverage metadata) such as GeoTIFF (http://trac.osgeo.org/geotiff/) or non-geo-referenced image formats, such as PNG, which are suitable for direct image display.

\section{EarthServer search engine and $x$ WCPS}

The EarthServer search engine builds on top of the above-mentioned technologies, offering a query language, an abstract data model, and a series of coordinating services. In particular, it offers the functionality to transparently exploit coverages distributed across different services. The main enabling component is a query language named 'xQuery compliant WCPS' or xWCPS (Kakaletris et al. 2013), which closely follows xQuery's syntax and philosophy allowing mixed search and results on both XML represented metadata and coverage data under a familiar syntactic formalism. An example of an xWCPS query that returns CRISM greyscale images with observation type 'FRT' combining both metadata and data follows:

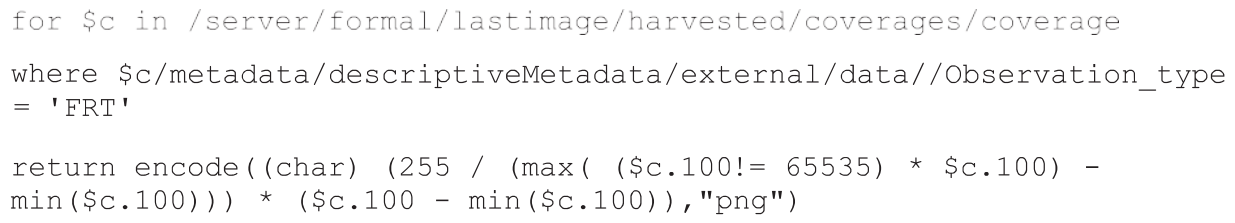

Listing 1: Example of an xWCPS query.

\section{Scalability}

One of the tasks of the EarthServer project was to enhance and prove scalability of the proposed technologies.

Generally, scalability of rasdaman, being an array DBMS, is leveraged through the following core properties, among others:

- by performing a partitioning of large arrays into tractable sub-arrays of suitable size. Suitability mainly is given by the access pattern to which the rasdaman arrays can be trimmed in the tiling clause of the insert statement. In the optimal case, a query can be answered with one disk access or entirely from cache. Categories of queries, so-called workloads, can be tuned this way. Examples include time series analysis where the incoming image slices are reshaped into time 'sticks.' In the extreme case, administrators provide only the location of hotspots in space and time; the systems will put these into single tiles for fast access and perform a suitable tiling around those by itself. Therefore, queries depend less on the size of the objects touched, but only on the size of the excerpt used from them.

- Whenever multiple tiles are loaded to answer a query, these can be processed in parallel, for example, in a multi-core environment. For objects sitting on different server nodes, parallel subqueries can be spawned.

- Query expressions can be replaced by more efficient ones. For example, adding two images pixelwise and then doing an average on the result image is less efficient than first averaging each image (which can be parallelized in addition) and 
then subtracting the two resulting single numbers. In rasdaman, every incoming query is analyzed against 150 rules to find the most efficient variant.

- On highest level, queries are optimized in various ways, including the methods listed, to achieve high performance. Such adaptive optimization is substantially more promising than the static parallelization methods deployed in techniques such as MapReduce. In rasdaman, query swarms can be distributed over a peer network of rasdaman-enabled servers ('inter-query parallelization'), and single queries can be split and distributed in the rasdaman network ('intra-query parallelization').

In the next sections we address both parallelization types and report tests conducted.

\section{Inter-query parallelization}

Inter-query parallelization spreads a large number of queries across different nodes. It is useful when executing a large number of small queries. For evaluation purposes, a set of tests were run performing 1000 queries over $\mathrm{N}$ slave virtual machines deployed on a 64 cores server, with $\mathrm{N}$ varying from 1 to 60 . Results demonstrated that while the average number of queries processed by each slave node decreases as $1 / \mathrm{N}$, the overall number of parallel processes saturated rapidly and the overall run time value decreased almost linearly. The inter-query parallelization is currently available on both standard andenterprise edition of rasdaman.

\section{Intra-query parallelization}

Intra-query parallelization smartly splits a complex query into many different small queries sent to different nodes in a network of rasdaman peers. Query splitting and placement is done in a way that minimizes data transfer and maximizes parallel evaluation (Dumitru, Merticariu, and Baumann 2014). This approach is useful when dealing with very big and complex queries. For evaluation, a rasdaman federation has been deployed in the Amazon Elastic Cloud (EC2) environment. Test queries have been executed in a series of scenarios by varying the number of network nodes up to more than 1000 . The results indicate good scalability of the system, with processing speed growing almost linearly with the number of nodes. More information about the testing procedure, used data-sets and queries, as well as detailed results and their interpretation can be foundin Merticariu's (2014) study.

\section{Ingestion}

At the beginning of the EarthServer project, the COMETA Grid infrastructure (laconoManno et al. 2010) was used to aid the processing and ingestion phase of the Planetary Service (see below Planetary Service section). To accomplish this task, a new Grid virtual organization (VO) 'vo.earthserver.eu' was created and registered in the official European Grid Infrastructure. Then a set of services and grid applications were developed, dealing with the overall processing in two separate phases. In the first phase, more than 7100 files containing input data for a total of about 0.5 TB were downloaded from NASA data archives by several grid jobs, piloted by the COMETA' Computing Elements and then stored on the COMETA' Grid Storage Elements. Each stored file to process was enriched with metadata from the AMGA metadata catalog (ARDA Project 2012). In the second phase, grid jobs were prepared and executed on the COMETA Grid sites to retrieve data 
according to their metadata, apply transformation algorithms to them, and store the outputback on the COMETA Storage Elements.

Up to 100 worker nodes were allocated to execute the jobs on the grid and the scaling factor measured was proportional to the number of worker nodes allocated, with a constant of proportionality close to 1 (the difference is due to the contribution of the job submission time to the total job execution time). Overall, almost 30,000 output files, organized in about 900 directories, have been produced for a total of about 6 TB of data.

\section{Visualization}

The service endpoints, offering coverages via WCS and WCPS, can be directly accessed over the web by any compatible client. To make the archives accessible in a more userfriendly and domain-specific way, data services provide a dedicated Web client interface, which builds queries according to user-specified parameter and displays results in a graphical form readily interpretable by the user. Both $2 \mathrm{D}$ widget and $3 \mathrm{D}$ visualization libraries have been made available for client-side interface development. The use of such client interfaces makes it as immediate as possible for users to interact with the contents of the data service archives, including map and graph display of aggregated data resultingfrom queries.

\section{$3 D$ client}

The EarthServer 3D web client builds on X3DOM (X3DOM, n.d.), an open-source framework and runtime for declarative 3D content. The 3D client deeply leverages the advantage of EarthServer technology: large datasets are split and accessed as smaller chunks and separately inserted into the browser Document Object Model (DOM) to maintain a high frame rate, thus enabling interactivity.

The client accesses data through OGC protocols like WCPS, WMS, and WCS (Herzig et al. 2013). Various modules turn data into visual representations, and multiple representations are combined in a shared 3D scene. In particular, WCPS, allowing extremely expressive queries, allowed enabling advanced client functionalities such as specifying different kinds of information for each RGB and alpha channel.

Additional visualization modules exist for point clouds (LIDAR), underground (SHARAD ground-penetrating radar) data, etc. Other features include annotations, axis labels, grids, exaggerations, separation of layers, etc. The outcome demonstrates that high-quality, hardware-accelerated, and flexible visualization of multidimensional data can be realized on the web by combining EarthServer server-side technology and X3DOM client-side technology (Figure 2).

\section{Mobile client}

EarthServer provides a mobile application named 'EarthServer SG Mobile' built for the two main platforms: Android (https://play.google.com/store/apps/details?id=it.infn.ct. earthserverSGmobile) and iOS (https://itunes.apple.com/us/app/earthserver-sgmobile/ id740603213? ls=1\&mt=8). The app provides access to a collection of three services (Figure 3):

(1) Access to Climate Data Services provided by the MEEO WCS server. The user can access a 97-hour forecast, as graph or image animation, since the selected date for a location specified or retrieved through the GPS. 


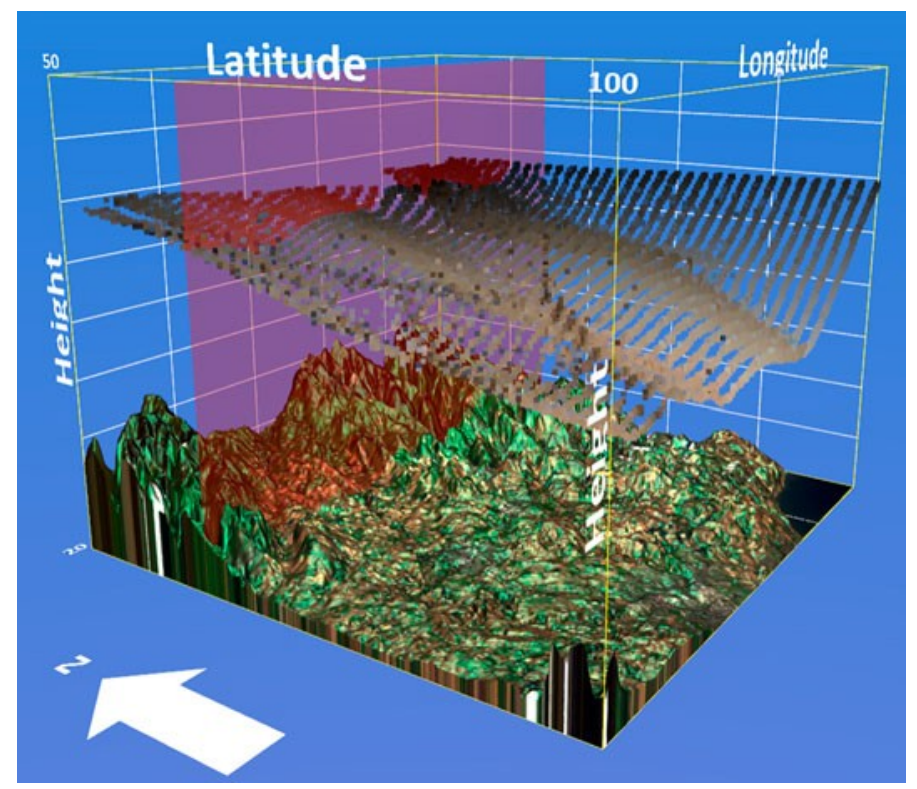

Figure 2. The 3D web client, configured to display data from two sources (DEM and point cloud) in a shared scene.

(2) A generic full WCS and WMS client, including visualization capabilities, developed by Software Engineering Italia. It shows coverages and map layers supporting user interaction.

(3) A repository browser of atmospheric data coming from the ESA MERIS spectrometer. The user can navigate the repository using a hierarchical filter mechanism based on asset metadata that allows users to find easily the queried assets.

The app supports authentication and authorization through the Catania Science Gateway Framework (Bruno and Barbera 2013) based on Shibboleth and LDAP technologies.

EarthServer in operation: the lighthouse applications

In order to demonstrate and validate the EarthServer technological solutions, six lighthouse applications have been developed. Five of them address specific science community needs, while the sixth one is a joint activity with NASA on secure access to data archives.

\section{Marine service}

The term 'Marine community' covers an extremely broad and diverse group including research scientists, commercial entities, and citizen scientists. In the past these groups used relatively small datasets, for instance, in situ collection of species presence or chlorophyll concentration. These discrete measurements have grown into extensive time series which are important in providing insight and context to complex scenarios such as climate change and species adaptation. 

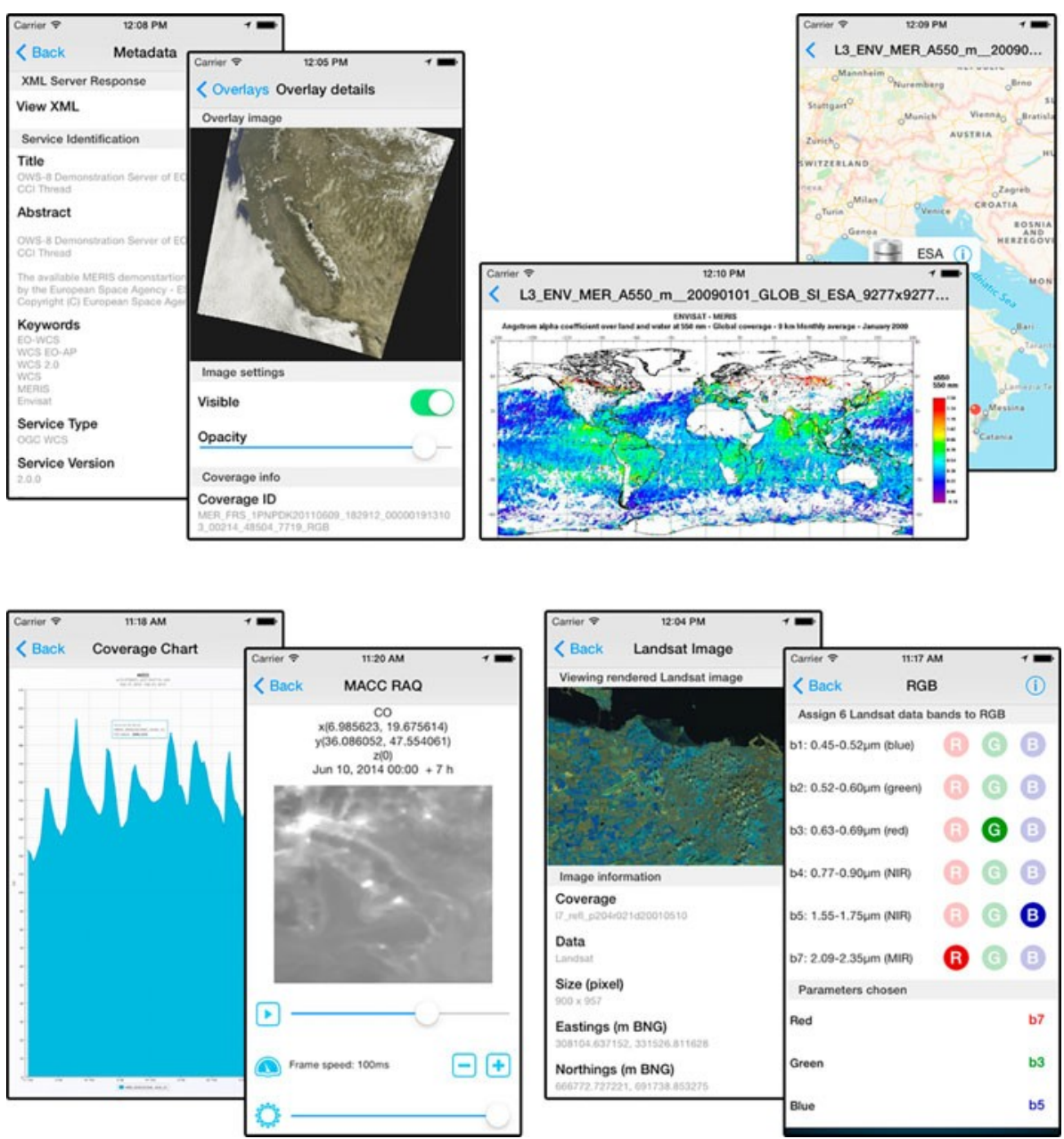

Figure 3. Screenshots from the EarthServer Science Gateway Mobile app.

With the advent and proliferation of remotely sensed data, the creation of larger and larger datasets has become common place in the marine community. Data are now at high spatial resolutions and available for decadal time series lengths. A single variable time series for the North Atlantic could be over 1 TB in size. This creates challenges for storage, transfer, and analysis.

The dataset selected as the core of the Ocean Data Service (PML 2013a) is the ESA Ocean Colour Climate Change Initiative (OC-CCl) chlorophyll time series (Clements and Walker 2013). This global dataset covers a 15-year time series (1998-present) created by merging the products of three sensors (SeaWiFS, MODIS, MERIS) and is around 17 TB in size). One reason for selecting the $\mathrm{OC}-\mathrm{CCl}$ dataset is that, as well as the directly sensed and indirectly computed parameters, the dataset contains per pixel metadata describing which sensors contributed to the parameter, a nine-class water type classification and two uncertainty estimates for each pixel. Few other ocean color datasets have such an extensive range of per pixel metadata and this provides a great opportunity to demonstrate 
how a more intelligent data service can be used to generate derived products based on combining these parameters into a single product in real time.

When creating the Ocean Data Service, the focus was on providing the user with the ability to interact with and analyze these large time series of remote-sensed data using a web-based interface. Data of interest may be selected using a graphical bounding box, and a simple 'timeline' has been implemented to allow sections of the time series to be selected using a similar paradigm. This geo-temporal range can then be used for analysis and visualization or data selection for download.

Giving users the ability to take the raw light reflectance data and use them to produce novel derived data products was also a key goal. To achieve this, a web-based band ratio client was created that allows users to drag and drop variables and mathematical operations (see Figure 4). Using this interface, users can replicate existing product creation algorithms or design and test new ones. The output of the algorithm is shown live allowing the user to make small adjustments and see how they affect the output.

Future work will see more plotting options for uses of the Ocean Data Service and a lifetime beyond the end of the EarthServer project. The ability to save and share analysis will be added creating a collaborative tool for exploration and analysis of big data products. The band ratio client will also be improved with a greater number of predefined mathematical operations, giving the user even more power to create and test novel band ratio algorithms.

\section{Geology service}

The EarthServer Geology Service (BGS 2013) has been developed by BGS (Laxton et al. 2013). The geosciences community is characterized by its diversity, with many subdisciplines each with different data and processing requirements. In developing the geology lighthouse service, the objective was to provide a valuable service for selected sub-disciplines, while at the same time to illustrate the potential of EarthServer

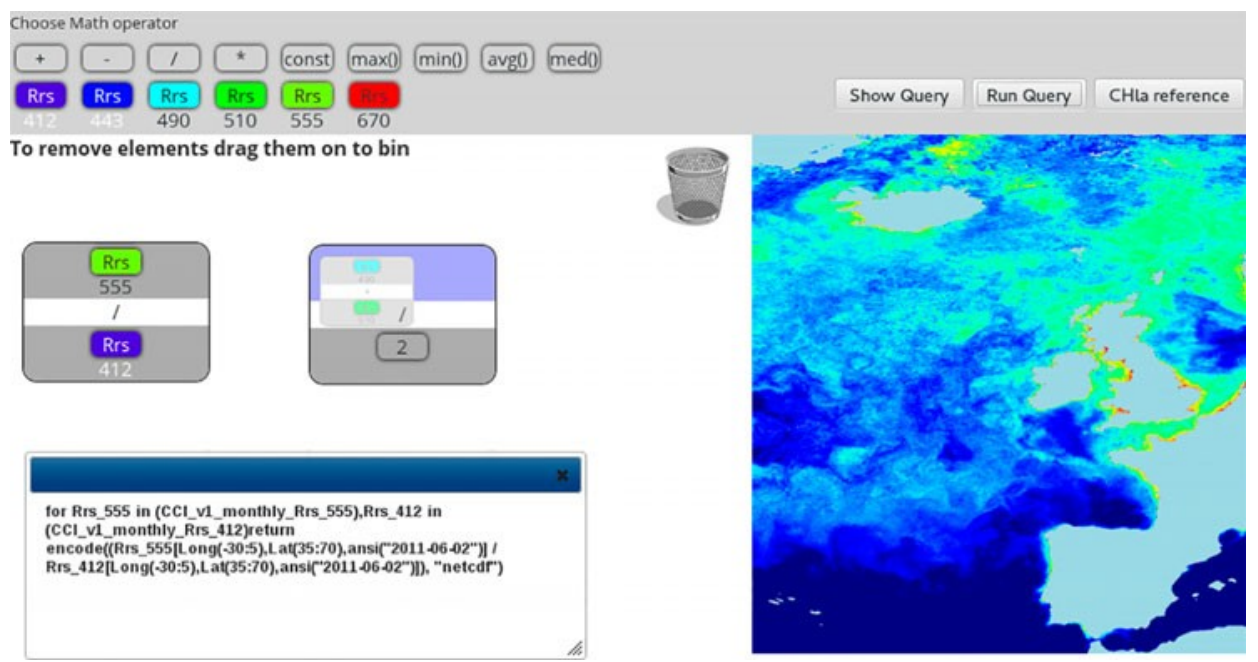

Figure 4. EarthServer Marine Service: Band Ratio Client provides a simple user interface to generate complex algorithms for teaching and testing new ideas. 
technology to the community as a whole. Geological remote sensing and 3D spatial modeling were chosen as the two areas that the service would concentrate on.

We carried out a survey of geosciences data providers (Mazzetti et al. 2012) and established that most geosciences sub-disciplines have limited experience in the use of coverage data, although the application of remotely sensed data to geoscience is well established and large data holdings have been built up by many geosciences organizations (Gupta 2003). Providing easy access to these data holdings, along with the ability to preview datasets for their suitability and carry out some simple pre-processing prior to download is the use case that the service aims to address.

Traditionally, geological maps were the principal means by which geological information was disseminated, and in recent times this has developed into the provision of digital maps and web services. There is an increasing move from geological maps to geological 3D spatial models, to make explicit the implicit geometry on geological maps (Howard et al. 2009). There is a requirement to deliver and visualize 3D models using web services and standard browsers (Mazzetti et al. 2012) and the EarthServer geology service aimed to address this with particular reference to the 3D spatial models of the superficial deposits of the Glasgow area (Monaghan et al. 2014). These models comprise 35 gridded surfaces separating geological units, each geological unit having an upper and a lower bounding surface. The remote sensed data available in the service comprises six band Landsat 7 (Blue, Green, Red, NIR 1, NIR 2, MIR) and three band false color aerial photography (NIR, green, blue) for the UK. The availability of digital terrain models (DTMs) is important for visualizing and processing both remotesensed data and models, and the service includes $50 \mathrm{~m}$ and $2 \mathrm{~m}$ resolution DTMs for the $U K$. By the end of the project, the combined data will have a volume of 20 TB.

It is a common feature of geosciences data that differing access constraints apply to different datasets. For example, the Landsat data in the geology service can be made freely available, whereas the aerial photographic data are restricted to use by BGS staff only. Two models of the superficial deposits of Glasgow were developed, one limited to central Glasgow and freely available and another covering a wider area and incorporating more data which are restricted to use by members of a user consortium who have signed a licence agreement. In order to address these differing access requirements, parallel services have been set up with GUls providing access to different sets of coverages.

The remote-sensed data is accessible through a web GUI which allows spatial selection to be carried out graphically against a range of background maps. The images available in the chosen area are listed and can be viewed, compared, and overlaid on DTMs in the 3D client to aid selection and download. The images can also be enhanced through interactive contrast adjustment (Figure 5) and the enhanced image downloaded. The GUI also provides access to the Glasgow model in the 3D client, where the user can view the model interactively, turn on and off individual surfaces, and increase the verticalexaggeration to enhance the geological features.

Future developments of the service will increase the range of data types available and move from specific EarthServer interfaces to incorporating coverage services into a rangeof domain-specific portals and applications.

\section{Atmospheric service}

The Climate Data Service is the lighthouse application developed by MEEO (MEEO 2013) to support the Climate and Atmospheric communities in exploiting the 


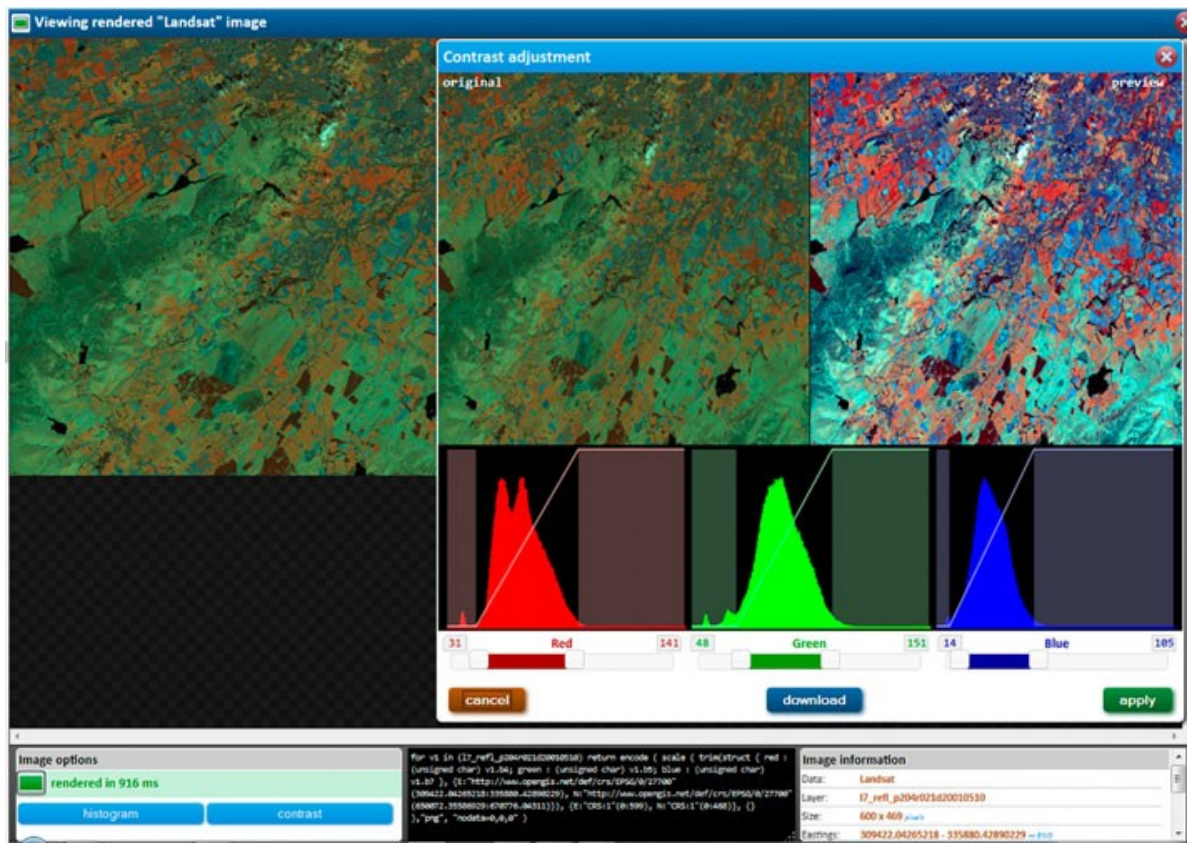

Figure 5. EarthServer Geology Service: Contrast adjustment of selected image.

heterogeneous multidimensional datasets at regional, European, and global scales (up to 5D: $\mathrm{x} / \mathrm{y} / \mathrm{z} /$ time/forecast time, in case of time series of forecasts of three-dimensional pollutant fields; Mantovani, Barboni and Natali 2013).

The Climate Data Service User Interest Group includes national and international authorities that use a variety of data to analyze environmental phenomena occurring at the different scales numerical model data, Earth Observation satellite products, ground measurements of atmospheric and meteo-climatic parameters are used independently (for specific applications, e.g. air quality monitoring) or simultaneously to improve operational products as in the case of assimilation of ground measurements and EO products into numerical models to improve air quality forecasts (Hirtl et al. 2014).

The common thread is the handling of big data variety and volumes: the expertise of the involved communities to manipulate Big Data is already consolidated, with hundreds of gigabytes of data processed and produced every day in operational and research processing chains. Nevertheless, the use of standard interfaces and services for real-time data manipulation, visualization, and exploitation are still not effective, leaving to offline processing components the role of performing data analysis and providing summary maps(e.g. three-hourly bulletins).

The Climate Data Service enables immediate access and processing capabilities to terabytes of data: the Multi-sensor Evolution Analysis (MEA) graphical user interface provides effective time series data analytic tools powered with WCS/WCPS interface offered by the rasdaman array database (ESA 2014). A powerful infrastructure $(+50$ CPUs, +150 GB RAM, and +20 TB disk space) distributed between the European Space Agency and MEEO allows real-time data exploitation on: 
(1) pixel basis: permitting the selection of a set of products to be simultaneously visualized and analyzed to investigate relationship of natural and anthropogenic phenomena (see Figure 6);

(2) area of interest basis: permitting the selection of limited or global areas analysis domains, to superimpose different measurements of the same parameter from different sources, or to drive the analysis of specific pixels providing a meaningful background map (see Figure 6).

As requested by the Atmospheric Science communities, the Climate Data Service has been enriched with 3D/4D/5D model-derived datasets (e.g. meteorological fields, pollutants maps, etc.) to allow the users implementing advanced access processing services via WCPS interface (http://earthserver.services.meeo.it/tools/\#wcps) - e.g. evaluating cross-comparison of satellite and model data, extract statistical parameters, etc. At present, more than 100 collections, including third-party data (aerosol optical maps over the entire globe, provided by ECMWF; meteorological fields and pollutants concentrations maps over Europe, Italy and Austria, provided by the SHMI, ENEA, and ZAMG, respectively) are available for intensive data exploitation.

By the end of the EarthServer project, the Climate Data Service is providing access and processing access to over 130 TB of ESA, NASA, and third-party products

\section{Cryospheric service}

The Cryospheric Service (EOX 2013) is designed to help the community discover and assess snow cover products (Ungar 2013). It consists of EarthServer infrastructure in the background, a synchronization tool between CryoService and the snow cover data provider. Furthermore, it is a web client which allows data preprocessing and visualization.

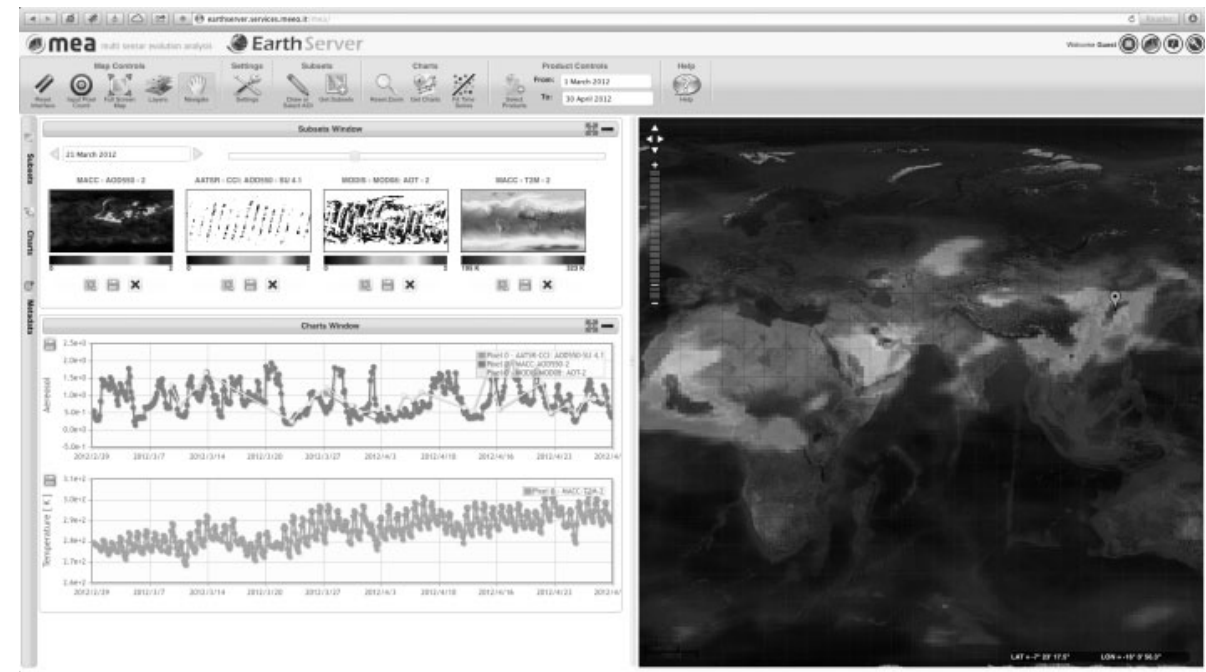

Figure 6. EarthServer Atmospheric Service: Analysis of aerosol (MACC, MODIS, AATSR) and temperature (MACC) time series: the AOT anomaly (high values) over China on 21 March 2013 is investigated to identify spatial-temporal impacts of meteorological parameters. 
The snow cover products are provided by the FP7 project Cryoland (http://cryoland. eu). The most relevant products are the Fractional Snow Cover (FSC) as well as the Snow Water Equivalent (SWE). Apart from pan-European coverages, CryoLand also produces regional products with a higher resolution which are accessible via the Cryospheric Service.

Additional data such as various digital elevation models (GTOPO30, SRTM, EU- DEM) and river basin districts (EEA WISE WFD) are available as well. This makes it possible to combine snow cover data with contour information and aggregating statistics over certain watershed areas. Watershed areas or drainage basins define areas of land where all surface water from precipitation converges into one single point, mostly a river. Estimating the amount of snow in such a watershed area therefore provides usefulinformation for hydrologists or hydroelectric power plants (Figure 7).

Snow products are mainly generated on a daily basis either from optical MODIS/Terra or from a combination of a satellite-based radiometer (DMSP SSM/I from 1987 to present) and snow depth data provided by ground-based weather stations (ECMWF). A synchronization script daily checks the availability of new time slices, downloads, ingests, and registers them into the EarthServer infrastructure.

Apart from the mandatory WMS, WCS, and WCPS endpoints (provided by rasdaman), the Cryospheric Service offers EO-WCS (Baumann and Meissl 2010) and EOWMS (Lankester 2009) endpoints (provided by EOxServer) to the data. The EO application profile for WCS is an extension designed to cope with specific needs of Earth Observation data. It adds mandatory metadata like time stamps and footprints as well as extended coverage definitions to represent dataset series or stitched mosaics.

The web application in the front end offers a map interface and two types of widgets to interact with the data. Selection widgets keep track of user-defined selections through the manipulation of the underlying models. Visualization widgets allow rendering of various graphs through heavy use of the D3 JavaScript library (D3 2013). The graphs are rendered using the responses of dynamically generated WCPS queries which are based onthe underlying model data.

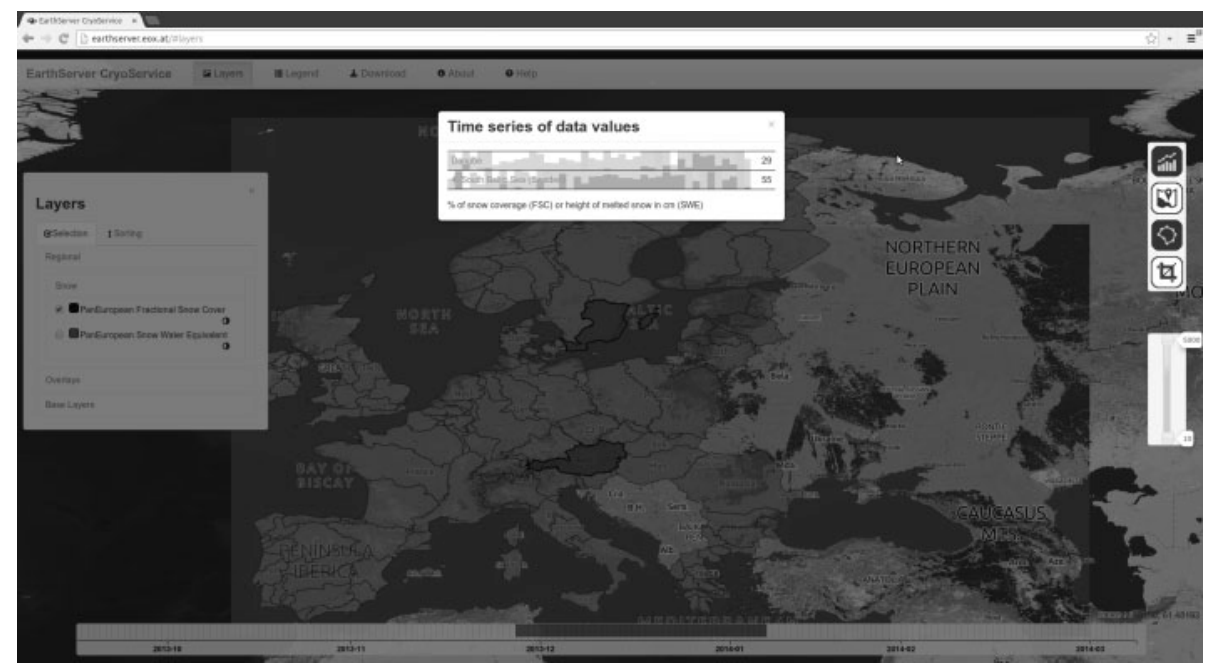

Figure 7. EarthServer Cryosphere Service: drainage basin statistics. 
Per product the user can select various spatial subsets: points of interests, river basin districts, or bounding boxes. In addition, subsets can be made according to the contour levels making use of the underlying digital elevation models. To define the temporal subset, a time slider widget was developed. Here, the user can select the time of interest which is then applied to the WCPS query. In addition, the time slider serves as well as a visualization tool for the respective product's temporal distribution.

In the end, the results are visualized via Hovmoeller-like diagrams (Hovmöller 1949) which are well suited to show time series of data coming from various locations, using the Cubism D3 plugin (Square 2012). This enables the user to assess the development of various snow cover parameters already aggregated on relevant spatial entities like river basin areas.

\section{Planetary service}

Planetary data are freely available on relevant archives provided by space agencies, such as the NASA Planetary Data System (PDS; McMahon 1996) and the ESA Planetary Science Archive (PSA; Heather et al. 2013) archives. Their exploitation by the community is limited by the variable amount of calibrated/higher level datasets. The complexity of these multi-experiment, multi-mission datasets is due largely to the heterogeneity of data themselves, rather than their sheer volume.

Orbital - so far - data are best suited for an inclusion in array databases. Most lander- or rover-based remote sensing experiment (and possibly in situ as well) are suitable for similar approaches, although the complexity of coordinate reference systems (CRS) is higher in the latter case.

PlanetServer, the Planetary Service of EarthServer (PlanetServer Home Page 2013) is a state-of-art online data exploration and analysis system based on the Open Geospatial Consortium (OGC) standards for Mars orbital data. It provides access to topographic, panchromatic, multispectral, and hyperspectral calibrated data. It has been under development at Jacobs University Bremen since October 2011 (Oosthoek et al. 2013). From the beginning of 2013, Software Engineering Italia provided refactoring and restyling of Planetary Service Client toward a Web 2.0-based application, namely the neoversion, followed by further developments.

Hyperspectral data from Mars currently can only be analyzed offline with relatively cumbersome processing and need to access commercial tools, in addition to the need for open source desktop/workstation data processing/reduction tools. WCPS allows for online analysis with a minimal fraction of the bandwidth and storage space needed by typical planetary image analysis workflows. The service focuses mainly on data from the Compact Reconnaissance Imaging Spectrometer (CRISM; Murchie et al. 2007) on board the NASA Mars Reconnaissance Orbiter (MRO). It does also include other imaging data, such as the MRO Context Imager (CTX) camera (Malin et al. 2007) and Mars Express (MEX) High Resolution Stereo Camera (HRSC; Jaumann et al. 2007; Gwinner et al. 2010), among others.

While its core focus has been on hyperspectral data analysis through the WCPS (Oosthoek et al. 2013; Rossi and Oosthoek 2013) matched to WMS map delivery, the service progressively expanded to host also subsurface sounding radar data (Cantini et al. 2014). Additionally, both single swath and mosaicked imagery and topographic data were added to the service, deriving from the HRSC experiment (Jaumann et al. 2007; Gwinneret al. 2010). 
The current Mars-centric focus can be extended to other planetary bodies and most components are general purpose ones, making possible its application to the Moon, Mercury, or alike.

The Planetary Service of EarthServer is accessible on http://www.planetserver.eu/, inaddition to the links to earthserver.eu project portal.

PlanetServer architecture comprises a main server (Figure 8a) and multiple Web Clients (Figure $8 \mathrm{~b}$ and $\mathrm{c}$ ). Further desktop GIS client efforts are moving forward. Both web clients are using standard web technologies and all the code developed within the
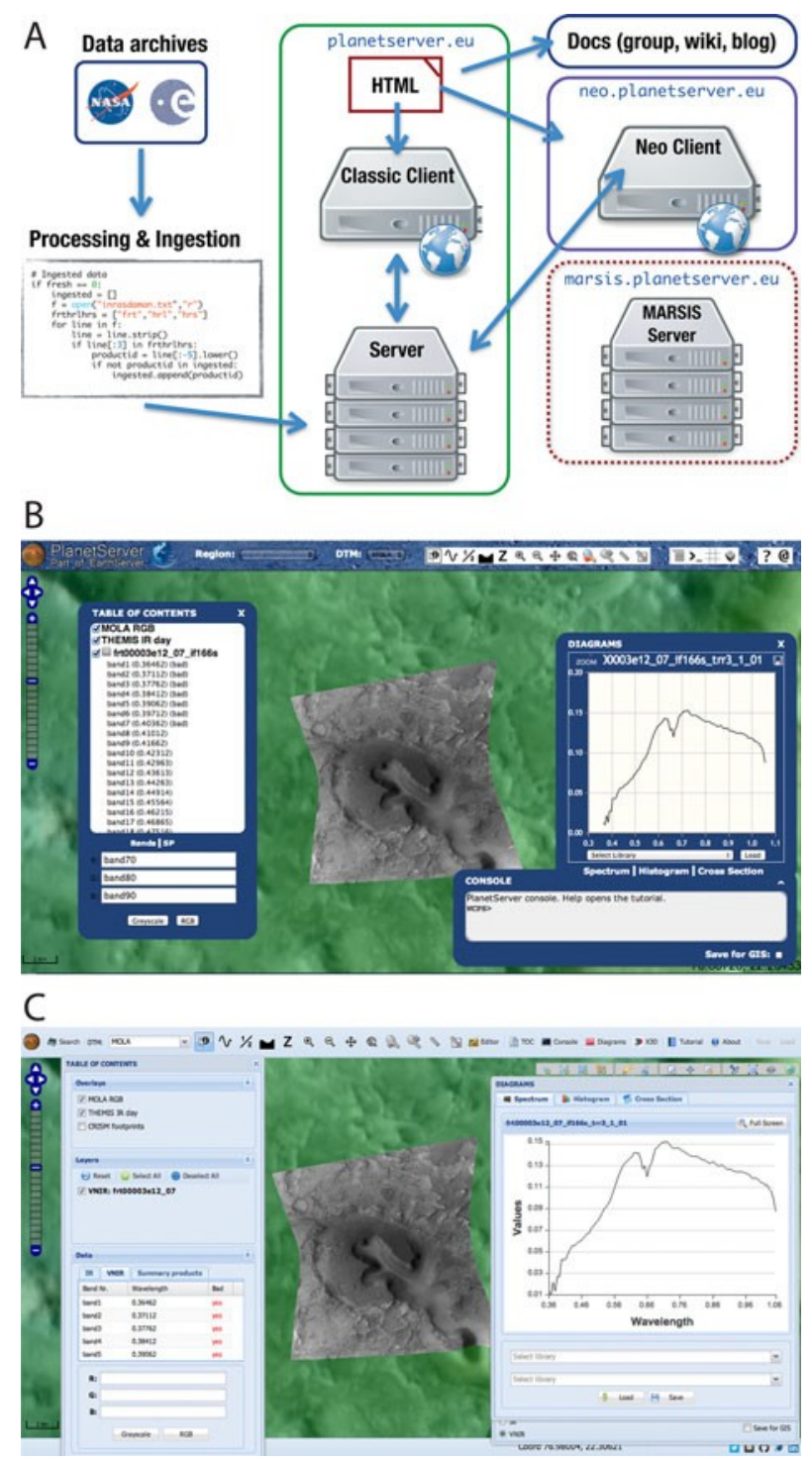

Figure 8. EarthServer Planetary Service: architecture (A) and multiple clients (B, C) and server setup. Original data derive from public Planetary Data System archives. Updated info and access onhttp://planetserver.eu. 
project is released on both the project repository and on GitHub on: https://github.com/ planetserver.

\section{Secured intercontinental access}

Many scientists working on NASA's Airborne Science programs share common dilemmas with their colleagues from ESA, and elsewhere on the planet. Datasets collected are large, and often cyclically acquired over the same areas. International collaborations are common, so participating scientists are identified and authenticated by discontinuous realms.

To achieve effective and expeditious results, both dilemmas had to be simultaneouslyaddressed.

A team with members from both the USA and EU assembled a secure, distributed, Identity Management and Service Provisioning system. This system utilizes open standards developed under the Open Geospatial Consortium (OGC) and Organization for the Advancement of Structured Information Standards (OASIS) stewardship.

The identity and service provisioning installation at the NASA Ames Research Center utilizes a variety of software engines: rasdaman, developed at Jacobs University, Bremen, Germany; MapServer, originally developed at the University of Minnesota, USA; and a Shibboleth/SAML2-based Security Engine developed by Secure Dimensions, Munich, Germany.

Recently demonstrated at the GEO-X Plenary in Geneva, the resulting installation, a participant in the EU FP7 Cobweb project, allows a user authenticated by any of the participants to access data services provided by any of the participants.

The scenario demonstrated in Geneva involved a user, authenticated by EDINA at the University of Edinburgh, who then accessed the NASA server for Unmanned Aircraft System (UAS) acquired wildfire imagery, the $E U$ server for radar-based elevation data, and combined them in real time, in a browser, to form a 3D landscape.

\section{Standardization impact}

EarthServer not only utilizes OGC standards in a rigorous, comprehensive manner, the project also has direct impact on the development of standards, thereby feeding back experience from the large-scale deployments done with the Lighthouse Applications.

\section{OGC}

We discuss three main areas addressed: advancing the OGC coverage data and service standards, combining coverages with GeoSciML, and mapping multidimensional coverages to the NetCDF data exchange format.

An online demonstrator (http://standards.rasdaman.org) has been established to explain OGC WCS, and WCPS use and to promote their uptake by geoservice stakeholders. Further, EarthServer has set up information resources on Wikipedia (http:// en.wikipedia.org/wiki/Coverage_data, http://en.wikipedia.org/wiki/Web_Coverage_Service, http://en.wikipedia.org/wiki/Web_Coverage_Processing_Service) and an OGC external wiki. 
A coverage, introduced in OGC Abstract Topic 6 (OGC 2007) and ISO 19123 (ISO 2005) is the general concept for space-time-varying data, such as regular and irregular grids, point clouds, and general meshes. Being high-level and abstract, however, these standards are not suitable for interoperable data exchange, and they also largely lack practice-oriented service functionality.

OGCs concrete coverage concept remedies this. A concise data definition of coverages is provided with GMLCOV (Baumann 2012a), allowing conformance testing of data and services down to single pixel level. GMLCOV relies on GML for a description that can be validated by a machine, but coverages by no means are constrained to that: any suitable data format - such as JPEG, NetCDF, comma-separated

values (CSV) - can be used as well. OGC format profiles define the mapping for each such format. The concrete definition of a coverage, together with a format encoding specification, allow instances to be created and transmitted among different software elements, thus ensuring interoperability: a key aspect to be achieved in modern archives to foster data dissemination and exploitation.

Any OGC service interface that can handle features - and, as such, the special case of coverages - can also offer coverages, for example, WFS, WPS, and SOS. However, the

dedicated WCS suite offers the most functionality (Baumann 2012b). The WCS core defines how to access and download a coverage or a cutout of it in some chosen format. WCS extensions add bespoke functionality like band ('channel', 'variable') extraction, scaling, and processing. They also define communication protocols over which services can be addressed, such as GET/KVP, SOAP, and (in future) REST and JSON. A specific facet is the Web Coverage Processing Service (WCPS) standard which adds an agile analytics language for spatio-temporal sensor, image, simulation, and statistics data. Figure 9 shows the 'Big Picture' of the OGC coverage suite.

In EarthServer, substantial contributions have been made to this framework. Several extension specifications have been established by Jacobs University and rasdaman $\mathrm{GmbH}$, most of them being implemented in rasdaman. Logically, rasdaman has become the OGC

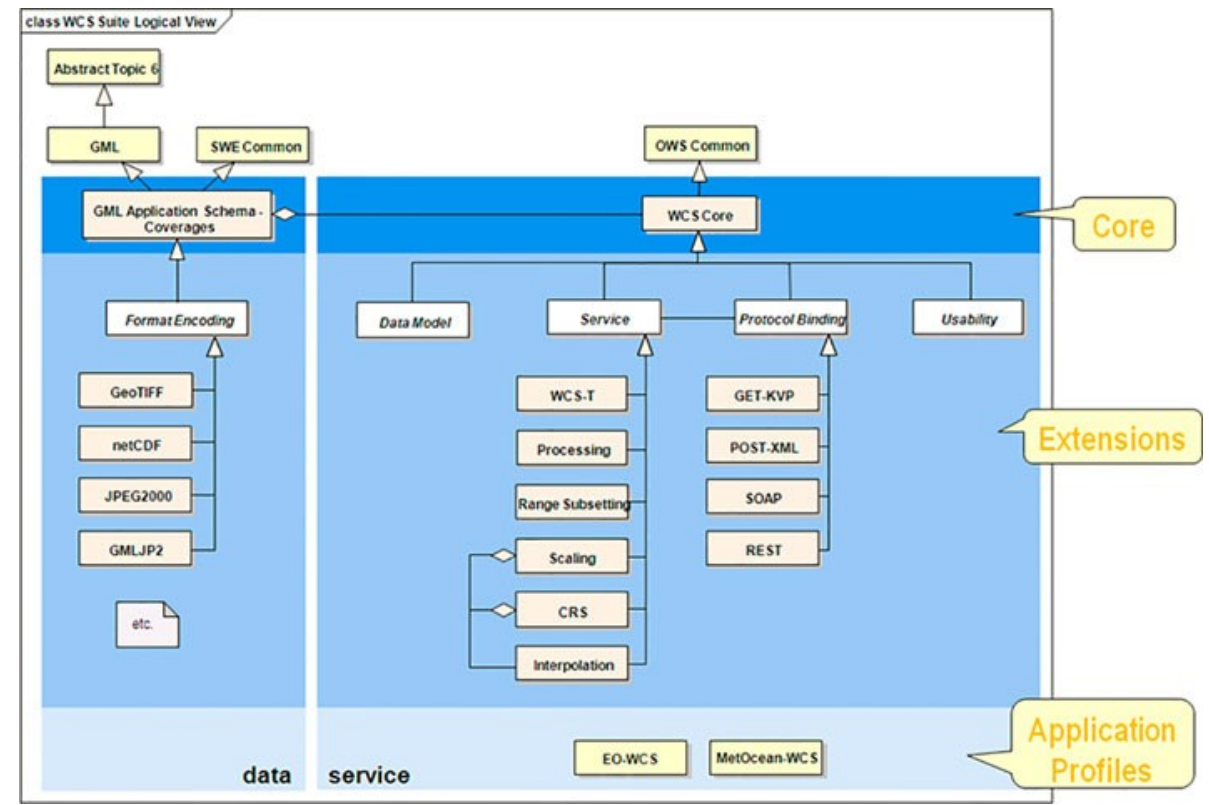


Figure 9. The OGC coverage suite of standards for spatio-temporal data access.

WCS Core Reference Implementation. The conformance tests required to check implementations have been developed and given to OGC for free use by all implementers.

Further, EarthServer has significantly enhanced understanding of coverage data which are not regularly gridded (such as orthoimages or climate datasets). Some special cases of irregular grids have been described by GML 3.3, and EarthServer has complemented this to also cover any combination of the GML 3.3 grid types. Point clouds have been integrated in WCS, and with rasdaman for the first time grids and point clouds can be served over the same WCS service interface. General meshes are being studied conceptually, aiming at a uniform algebraic treatment and a query language (possibly extending WCPS) for such coverages.

A major conceptual issue that has arisen is the insufficient understanding of the time dimension. Traditionally, time axes have been treated independently and differently from spatial axes. EarthServer has worked hard to convince OGC of the need for uniform coordinate reference systems (CRSs) integrating space and time (Baumann et al. 2012). A particularly interesting use case is WCS slicing. Assuming a $4 \mathrm{D} x / \mathrm{y} / \mathrm{z} / \mathrm{t}$ coverage, users can obtain any axis subset through slicing. Most such combinations, such as $x / t$ and $y / z$, are not covered by traditional CRSs. Therefore, a mechanism has been devised which allows recombination of new CRSs and axes on the fly, based on the URL-based naming scheme used by OGC. A corresponding URL resolver, SECORE, has been implemented and is now in operational use by OGC (Misev, Rusu, and Baumann 2012).

\section{WCPS}

Basically, WCPS (P. Baumann, Web Coverage Processing Service (WCPS) Implementation Specification, OGC 08-068 2008) consists of a query language in the tradition of SQL, but targeted to spatio-temporal coverages and with specific geo-semantics support. The basic query schema consists of a for clause where an iteration variable is bound to a series of coverages to be inspected in turn. This 'loop' can be nested, thereby allowing combination of different datasets. In the return clause, an expression containing variable references is provided which the server evaluates. Scalar results are transmitted back in ASCII, coverage-valued results get encoded in some user-selected data format prior to sending. Optionally, a where clause can be added which acts as a filter on the coverages to be inspected. (Baumann 2010) provides an introductory overview, whereas PML (2013b) offers an online tutorial focusing on the Marine/Ocean domain.

The following example delivers 'From MODIS scenes ModisScene1, ModisS-cene2, and ModisScene3, the absolute of the difference between red and nir (near-infrared) bands, in NetCDF - but only for those where nir exceeds 127 somewhere within region $\mathrm{R}^{\prime}$ :

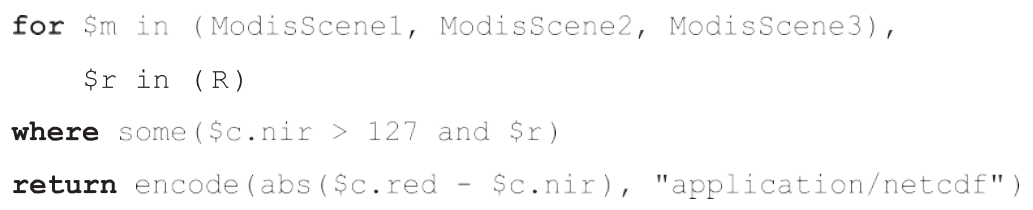


Listing 2: Sample WCPS query.

Readers familiar with XQuery will notice that the WCPS syntax is close to the standard XML query language XQuery (W3C 2014). This is intentional, as metadata in today's operational systems typically are represented in $\mathrm{XML}$, and the door shouldremain open for a later integration of both.

In order to include extended coverage notion and to incorporate new functionality requested by service operators in WCPS, WCPS 2.0 is under development by Jacobs University and rasdaman $\mathrm{GmbH}$, paired by implementation work of ATHENA (cf. Section 'EarthServer Search Engine and xWCPS'). It addresses: harmonization with GMLCOV and WCS, support for irregular grids, XQuery integration, invocation of external code (e.g. Hadoop) from within a query, an extended operation set, polygonal data extraction, etc.

\section{GeoSciML}

GeoSciML is a GML-based data transfer standard for geoscientific information which is typically shown on geological maps. The geological objects in 3D spatial models are the same as those on maps and we have investigated the use of GeoSciML to describe the models delivered by the EarthServer geology service. The objective is to enable queries against both the GeoSciML description and the coverage values such as 'find all geological units with a predominant sand lithology within $25 \mathrm{~m}$ of the surface'. GeoSciML was incorporated into the coverage metadata where it can be queried using xWCPS.

\section{NetCDF}

NetCDF is a set of software libraries and self-describing, machine-independent data formats that support the creation, access, and sharing of array-oriented scientific data (UCAR, n.d.). It is maintained by the UNIDATA Program at the University Corporation for Atmospheric Research (UCAR). As a data model, netCDF is able to accommodate multidimensional array data types. Specific semantics is provided through conventions; the most widespread convention is the Climate and Forecast convention (CF) widely adopted especially in the Meteo/Ocean community. CF-netCDF and related specifications are standardized by the OGC through the OGC CF-netCDF SWG (Domenico 2010, 2011). EarthServer contributed in the definition of specifications for the harmonization of ISO coverage types and the netCDF data model (Domenico 2012; Domenico and Nativi 2013).

ISO

The ISO SQL database query standard, while originally focusing on table-oriented structures, has seen several extensions to accommodate further relevant information structures. Recently, Jacobs University and rasdaman $\mathrm{GmbH}$ jointly proposed to ISO to add support for multidimensional arrays. As of its meeting in June 2014, ISO/IEC JTC1 WG3 has commenced activities on SQL/MDA (Multi-Dimensional Arrays).

\section{Related work}

Statistics and image processing tools like R and Matlab have supported processing array 
data types for a considerable time. More recently, desktop tools like the Analysis and Display System (GrADS, http://iges.org/grads/) have joined.

However, this class of engines is mostly limited to main memory and definitely not scalable to Petabyte sizes. Such scalability is provided with Array Databases (Baumann 2009). A more recent approach is SciQL (Kersten et al. 2011) which extends SQL with array operations, but with a different paradigm where arrays are treated like tables. This is not expected to scale to millions of arrays, such as satellite image archives. Further, SciQL does not yet have a scalable storage and query evaluation engine. SciDB (n.d.), even more recent, is following a similar approach to SciQL; only a lab prototype is known today, so scalability is still to be proven. Another class of systems is constrained to small 2D arrays; PostGIS Raster (Paragon, n.d.), Oracle GeoRaster (Oracle, n.d.), and Teradata arrays (Teradata, n.d.) fall into this category. Hence, rasdaman is the only Array Database operationally used on multi-TB holdings, fully parallelized, and proven in scalability.

OPeNDAP is a project developing the Hyrax server implementing the DAP (Data Access Protocol). Based on a procedural array language, Hyrax allows accessing data files on local or remote servers while abstracting from the data format, mainly centering around NetCDF. Since recently, OPeNDAP supports WCS as well, not yet WCPS, though. There is no evidence, or thought, that the (procedural, so harder to parallelize) OPeNDAP language is supported by adaptive storage organization and parallelization as the (declarative, so optimizable) WCPS query language is through rasdaman.

MapReduce is often mentioned in the context of Big Data due to its builtin parallelization support. However, MapReduce is not aware of the specific nature of arrays with the n-D Euclidean neighborhood of array cells - when once cell is used by the application it is extremely likely that its direct neighbors will be fetched soon after. Systems like rasdaman support this through adaptive tiling, MapReduce will do only splitting of a large array if programmed so by the user. Further, existing algorithms have to be rewritten into the $\operatorname{map}()$ and reduce() functions of this particular approach which requires significant effort and skills by the user. With WCPS, on the other hand, a highlevel language in the tradition of SQL where the engine determines at runtime and individually how to distribute load between nodes. Finally, MapReduce assumes a homogeneous operational interface - no case has been reported where requests have been distributed over independent data centers with heterogeneous hardware, as has been done with rasdaman in EarthServer where ad-hoc data fusion between a NASA and an ESA server has been demonstrated.

Aside from such general-purpose techniques, dedicated tools have been implemented. The NOAA Live Access Server (LAS, http://ferret.pmel.noaa.gov/LAS) is a highly configurable web server designed to provide flexible access to geo-referenced scientific data. Capabilities include access to distributed datasets, visualization capabilities, and connectors to common science tools. It seems, though, that LAS does not offer a flexible query language with internal parallelization. Also, it does not support any of the OGC 'Big Geo Data' standards WCS and WCPS.

\section{Conclusions}

The EarthServer infrastructure provides a fully functional, production-level set of flexible and interoperable data services, fully committed to the OGC coverage standards suite and the OGC WCPS query language that can be leveraged to effectively filter, extract, and process coverage data. Such data, depending on the chosen encoding, is suitable 
for immediate visualization or for further processing (e.g. making the services directly usable as data elements for a staged processor). This approach also avoids the need to locate, download and access data files in native format or the need to process them with specific (dataset dependent) tools or custom written programs, in favor of a flexible query language, over a unified data model. Finally, parameterization of a query is straightforward, thus enhancing client-side integration and ease of development.

These capabilities have been demonstrated in implementation of several large Lighthouse applications, each covering a specific Earth Sciences domain and bringing large datasets and differing community needs.

\section{Disclosure statement}

No potential conflict of interest was reported by the authors.

\section{Funding}

The research leading to these results has received funding from the European Community under grant agreement 283610 EarthServer.

\section{References}

Aiordachioaie, Andrei, and Peter Baumann. 2010. "PetaScope: An Open-source Implementation of the OGC WCS Geo Service Standards Suite." In Proceedings of the 22nd International Conference on Scientific and Statistical Database Management, edited by Michael, Gertz, and Bertram Ludäscher, 160-168. Heidelberg: Springer.

ARDA Project. 2012. AMGA - Overview. Aprile 16. http://amga.web.cern.ch/amga/.

"Array DBMS." 2014. Wikipedia, Agosto 20. http://en.wikipedia.org/wiki/Array_DBMS. Bargellini,

Pier, Cheli Simonetta, Yves-Louis Desnos, Bruno Greco, veronica Guidetti, Marchetti Pier Giorgio, Carmen Comparetto, Stefano Nativi, and Geoff Sawyer. 2013. Big Data from Space - Event Report. Frascati: ESA. http://www.congrexprojects.com/docs/default-source/ 13c10_docs/13c10_event_report.pdf?sfvrsn=2.

Baumann, Peter. 1994. "Management of Multidimensional Discrete Data." The VLDB Journal 3 (4): 401-444. doi:10.1007/BF01231603.

Baumann, Peter. 2008. Web Coverage Processing Service (WCPS) Implementation Specification, OGC 08-068. Version 1.0. OGC. http://www.opengeospatial.org/standards/.

Baumann, P. 2009. "Array Databases and Raster Data Management." In Encyclopedia of Database Systems, edited by L. Liu, and T. Özsu. Heidelberg: Springer. www.opengeospatial.org/ standards/.

Baumann, Peter. 2010. "The OGC Web Coverage Processing Service (WCPS) Standard." Geoinformatica 14 (4): 447-479. doi:10.1007/s10707-009-0087-2.

Baumann, Peter. 2012a. "OGC GML Application Schema - Coverages." OGC 09-146r2. Version 1.0. OGC.

Baumann, Peter. 2012b. “OGC ${ }^{\circledast}$ WCS 2.0 Interface Standard - Core." OGC 09-110r4. Version 2.0. OGC.

Baumann, Peter, Piero Campalani, Jinsongdi Yu, and Dimitar Misev. 2012. "Finding My CRS: A Systematic Way of Identifying CRSs." In Proceedings of the 20th International Conference on Advances in Geographic Information Systems, 71-78. Redondo Beach, CA: ACM, ISBN 978-14503-1697-2.

Baumann, Peter, Bill Howe, Kjell Orsborn, and Silvia Stefanova. 2011. "EDBT/ICDT Workshop on Array Databases." Proceedings of 2011 EDBT/ICDT Workshop on Array Databases, Uppsala, Sweden.

Baumann, Peter, and Stephan Meissl. 2010. OGC WCS 2.0 Application Profile Earth Observation. OGC 10-140. Version 1.0. OGC.

BGS. 2013. Geology Service Home Page. British Geological Survey. Accessed September 1, 2013. http://earthserver.bgs.ac.uk/. 
Bruno, Riccardo, and Roberto Barbera. 2013. "The EarthServer Project and Its Lighthouse Applications: Exploiting Science Gateways and Mobile Clients for Big Earth Data Analysis." In Proceedings International Symposium on Grids and Clouds (ISGC). Taipei: Academia Sinica.

Cantini, Federico, Angelo Pio Rossi, Roberto Orosei, Peter Baumann, Dimitar Misev, Jelmer Oosthoek, Alan Beccati, Piero Campalani, and Vikram Unnitan. 2014. MARSIS Data and Simulation Exploited Using Array Databases: PlanetServer/EarthServer for Sounding Radars. Vienna: Geophysical Research Abstracts.

Clements, Oliver, and Peter Walker. 2013. EarthServer Project Deliverable D240.12. Ply- mouth: PML.

D3. 2013. Data-Driven Documents. Accessed 2014. http://d3js.org/.

Domenico, Ben. 2010. OGC CF-netCDF Core and Extensions Primer. OGC 10-091r1. Version 1.0. OGC.

Domenico, Ben. 2011. OGC Network Common Data form (NetCDF) Core Encoding Standard Version 1.0. OGC. http://portal.opengeospatial.org/files/?artifact_id=43732.

Domenico, Ben. 2012. OGC Network Common Data form (NetCDF) NetCDF Enhanced Data Model Extension Standard. OGC. https://portal.opengeospatial.org/files/?artifact_id=50294.

Domenico, Ben, and Stefano Nativi. 2013. CF-netCDF3 Data Model Extension Standard. OGC. https://portal.opengeospatial.org/files/?artifact_id=51908.

Dumitru, A., V. Merticariu, P. Baumann. 2014. Exploring Cloud Opportunities from an Array Database Perspective. Snowbird, USA: Proceeding of the ACM SIGMOD Workshop on Data analytics in the Cloud (DanaC'2014).

EOX. 2013. CryoService Home Page. EOX. Accessed September 1, 2013. http://earthserver.eox.at.

ESA. 2014. Multi-sensor Evolution Analysis. 28 Aprile. http://wiki.services.eoportal.org/tiki-index. php?page=Multi-sensor+Evolution+Analysis.

Gupta, R. P. 2003. Remote Sensing Geology. Berlin: Springer.

Gwinner, Klaus, Frank Scholten, Frank Preusker, Stephan Elgner, Thomas Roatsch, Michael Spiegel, Ralph Schmidt, Jurgen Oberst, Ralf Jaumann, and Christian Heipke. 2010. "Topography of Mars from Global Mapping by HRSC High-resolution Digital Terrain Models and Orthoimages: Characteristics and Performance." Earth and Planetary Science Letters 294 (3): 506-519. doi:10.1016/j.epsl.2009.11.007.

Heather, David J, Maud Barthelemy, Nicolas Manaud, Santa Martinez, Marek Szumlas, Jose Luis Vazquez, and Pedro Osuna. 2013. "ESA's Planetary Science Archive: Status, Activities and Plans." European Planetary Science Congress. http://www.epsc2013.eu/.

Herzig, Pasquale, Michael Englert, Sebastian Wagner, and Yvonne Jung. 2013. "X3D-EarthBrowser: Visualize Our Earth in Your Web Browser." Web3D'13 Proceedings of the 18th International Conference on 3D Web Technology. San Sebastian: ACM New York.

Hey, Tony, and Anne E. Trefethen. 2002. "The UK e-Science Core Programme and the Grid." International Journal for e-Learning Security (IJeLS) 1 (1/2): 1017-1031.

Hirtl, Marcus, Simone Mantovani, Bernd C. Krüger, Gerhard Triebnig, Claudia Flandorfer, and Maurizio Bottoni. 2014. "Improvement of Air Quality Forecasts with Satellite and Ground Based Particulate Matter Observations." Atmospheric Environment 1: 20-27.

Houghton, Nigel. 2013. "ESA Reprocessing. A Service Based Approach." Big Data from Space. Frascati. http://www.congrexprojects.com/docs/default-source/13c10_docs/session-2a.zip.

Hovmöller, Ernest. 1949. "The Trough-and-Ridge Diagram." Tellus 1 (2): 62-66.

Howard, Andrew, Bill Hatton, Femke Reitsma, and Kenneth Lawrie. 2009. "Developing a Geoscience Knowledge Framework for a National Geological Survey Organisation." Computers and Geosciences 35 (4): 820-835.

lacono-Manno, Marcello, Marco Fargetta, Roberto Barbera, Alberto Falzone, Giuseppe Andronico, Salvatore Monforte, Annamaria Muoio, et al. 2010. "The Sicilian Grid Infrastructure for High Performance Computing." International Journal of Distributed Systems and Technologies 1: 4054.

ISO. 2005. Geographic Information - Schema for Coverage Geometry and Functions. ISO 19123:2005.

Jacobs University Bremen and Rasdaman GmbH. 2013. Rasdaman Project, Standards and Demonstrator Page. Accessed September 15, 2013. http://standards.rasdaman.org/.

Jaumann, Ralf, Gerhard Neukum, Thomas Behnke, Thomas C. Duxbury, Karin Eichentopf, Joachim Flohrer, Stephan van Gasselt, et al. 2007. "The High-resolution Stereo Camera (HRSC) 
Experiment on Mars Express: Instrument Aspects and Experiment Conduct from Interplanetary Cruise Through the Nominal Mission." Planetary and Space Science 55 (7): 928-952.

Kakaletris, George, Panagiota Koltsida, Thanassis Perperis, and Peter Baumann. 2013. EarthServer Project Deliverable, Query Language Pilot, D330.12. EarthServer Project.

Kersten, M., Y. Zhang, M. Ivanova, and N. Nes. 2011. "SciQL, a Query Language for Science Applications." Proceedings of the Workshop on Array Databases, Uppsala.

Laney, Doug. 2001. 3D Data Management. Controlling Data Volume, Velocity, and Variety in Application Delivery Strategy. Stamford: META Group.

Lankester, Thomas. 2009. OpenGIS Web Map Services - Profile for EO Products. OGC 07-063r1.

Laxton, John, Marcus Sen, James Passmore, and Simon Burden. 2013. EarthServer Project Deliverable D250.11, OGC 07-063r1. Nottingham: BGS.

Malin, Michael C., James F. Bell, Bruce A. Cantor, Michael A. Caplinger, Wendy M. Calvin, Robert Todd Clancy, Kenneth S. Edgett, et al. 2007. "Context Camera Investigation on Board the Mars Reconnaissance Orbiter." Journal of Geophysical Research: Planets 112: 2156-2202. doi:10.102 9/2006JE002808.

Mantovani, Simone, Damiano Barboni, and Stefano Natali. 2013. EarthServer Project Deliverable D230.12. Ferrara: MEEO.

Mazzetti, Paolo, Angelo Pio Rossi, Oliver Clements, Simone Mantovani, Stefano Natali, Maria Grazia Veratelli, Joachim Ungar, and John Laxton. 2013. Community Reporting. EarthServer Deliverable D120.21.

Mazzetti, Paolo, John Laxton, Maria Grazia Veratelli, and Mike Grant. 2012. Community Reporting. EarthServer Deliverable D120.21.

McMahon, Susan K. 1996. "Overview of the Planetary Data System." Planetary and Space Science 44 (1): 3-12.

MEEO. 2013. Climate Data Service Home Page. MEEO. Accessed September 1, 2013. http:// earthserver.services.meeo.it/.

Merticariu, George. 2014. "Keeping 1001 Nodes Busy." BSc diss., Bremen: Jacobs University.

Misev, Dimitar, Mihaela Rusu, and Peter Baumann. 2012. "A Semantic Resolver for Coordinate Reference Systems." Proceedings of 11th International Symposium on Web and Wireless Geographical Information Systems (W2GIS), 47-56. Naples: Springer.

Monaghan, A. A., S. L. B. Arkley, K. Whitbread, and M. McCormac. 2014. Clyde Superficial Deposits and Bedrock Models Released to the ASK Network 2014: A Guide for Users. Version 3. Nottingham: British Geological Survey.

Murchie, Scott L., Raymond E. Arvidson, Peter Bedini, K. Beisser, J. P. Bibring, J. Bishop, J. Boldt, et al. 2007. "Compact Reconnaissance Imaging Spectrometer for Mars (CRISM) on Mars Reconnaissance Orbiter (MRO)." Journal of Geophysical Research: Planets 112 (E5): 21562202. doi:10.1029/2006JE002682.

OGC. 2007. Abstract Specification Topic 6: Schema for Coverage Geometry and Functions. OGC 07-011. Version 7.0. OGC.

Oosthoek, J. H. P., J. Flahaut, A. P. Rossi, P. Baumann, D. Misev, P. Campalani, and V. Unnithan. 2013. "PlanetServer: Innovative Approaches for the Online Analysis of Hyperspectral." Advances in Space Research 23 (12): 1858-1871. doi:10.1016/j.asr.2013.07.002.

Oracle. n.d. Accessed June 10, 2014. http://www.oracle.com.

Paragon. n.d. PostGIS. Accessed June 10, 2014. http://www.postgis.org.

Petitdidier, Monique, Roberto Cossu, Paolo Mazzetti, Peter Fox, Horst Schwichtenberg, and Wim Som de Cerff. 2009. "Grid in Earth Sciences." Earth Science Informatics 2: 1-3.

PML. 2013a. Ocean Data Service Home Page. PML. Accessed September 1, 2013. http:// earthserver.pml.ac.uk/portal.

PML. 2013b. WCPS How to Pages (EarthServer: Ocean Data Service). Accessed September 14, 2013. http://earthserver.pml.ac.uk/portal/how_to/.

"Rasdaman." 2013. Wikipedia, December 29. http://en.wikipedia.org/wiki/Rasdaman.

Rasdaman GmbH. 2013. Rasdaman Query Language Guide. 8.4 ed., 91. Bremen: Rasdaman GmbH.

Rossi, Angelo Pio, and Jelmer Oosthoek. 2013. EarthServer Project Deliverable D260.12. Bremen: Jacobs University Bremen.

Sarawagi, Sunita, and Michael Stonebraker. 1994. "Efficient Organization of Large Multidimensional Arrays." Proceedings of the 11th IEEE ICDE Conference, Hannover, Germany, 328-336.

SciDB. n.d. SciDB. Accessed June 10, 2014. http://www.scidb.org. 
Square. 2012. Cubism.js. https://square.github.io/cubism/.

Teradata. n.d. Accessed June 10, 2014.

http://www.teradata.com.

UCAR. n.d. Network Common Data Form (NetCDF). Accessed March 27, 2014. http://www. unidata.ucar.edu/software/netcdf/.

Ungar, Joachim. 2013. EarthServer Project Deliverable D220.12. Wien: EOX.

W3C. 2014. "XQuery 3.0: An XML Query Language." http://www.w3.org/TR/2014/REC-xquery-3020140408/.

X3DOM. n.d. x3dom Homepage. http://www.x3dom.org. 